\title{
Cestodiasis tisulares: participación de los linfocitos T cooperadores 1 y 2
}

\author{
Héctor Samuel López-Moreno, Dr en C.(1)
}

\begin{abstract}
López-Moreno HS. Cestodiasis tisulares: participación de los linfocitosT cooperadores 1 y 2. Salud Publica Mex 2002;44:145-152. El texto completo en inglés de este artículo está disponible en: http://www.insp.mx/salud/index.html

\section{Resumen}

La cisticercosis y la hidatidosis son las parasito sis causadas por los metacéstodos de Taenia solium y de Echinococcus multilocularis (o E. granulosus), respectivamente. El estudio de las enfermedades parasitarias ofrece uno de los mejores modelos in vivo para el estudio de la división efectora de las subpoblaciones de linfocitos T cooperadores CD $4^{+}$, designadas Th1 y Th2 de acuerdo con el patrón de citocinas que producen. La polarización hacia alguno de estos fenotipos puede marcar la diferencia entre una respuesta inmune celular protectora o una respuesta inmune permisiva para la infección. La participación de los linfocitos Th1 y Th2 en las cestodiasis tisulares (cisticercosis e hidatidosis) ha sido estudiada en modelos experimentales de ratón y en pacientes humanos; en ambos casos los resultados sugieren que en los individuos con cisticercosis o hidatidosis la respuesta inmune celular está polarizada hacia un fenotipo Th2. En tanto que en los individuos do nde los parásitos son destruidos, o su desarrollo está limitado, la respuesta inmune celular se encuentra polarizada hacia un fenotipo Thl. El texto completo en ingles de este artículo está disponible en:http:/ /www.insp.mx/salud/index.html
\end{abstract}

Palabras clave: cisticercosis; equinococosis; linfocitosT auxiliadores-inductores 1 (Th1); linfocitosT auxiliadores-inductores 2 (Th2)

\section{López-Moreno HS.}

Tisular cestodiasis:The role

ofT helper cell subsets 1 and 2.

Salud Publica Mex 2002;44:145-152.

The English version of this paper

is available at: http://www.insp. mx/salud/index.html

\begin{abstract}
A bst ract
Cysticercosis and hydatidosis are parasitic diseases caused by larvae of Taenia solium and Echinococcus sp., respectively. Parasitic diseases are useful models for in vivo studies of effector functions of T helper cell subsets 1 and 2, (called Th1 and Th2 on the basis of the pattern of cytokines they produce). The polarization to Th1 or Th2 is related to protective or permissive immune responses in these diseases. The role of Th1 and Th2 lymphocytes in tissue cestodiasis (cysticercosis and hydatidosis) has been studied in experimentally infected mice and in human patients; study results suggest that in individuals with cysticercosis or hydatidosis, the cellular immune response is polarized to Th2, while individuals in whom parasites are killed or their growth is limited, have an immune cellular response that is polarized towards Th1. The English version of this paper is available at: http://www.insp.mx/salud/index.html
\end{abstract}

Key words: cysticercosis; echinococcosis; T-lymphocytes, helper-inducer subset 1 (Th1);T-lymphocytes, helper-inducer subset 2 (Th2)

(1) Departamento de Biomedicina Molecular, Centro de Investigación y de Estudios Avanzados del Instituto Politécnico N acional. México, D.F., México.

Fecha de recibido: 29 de enero de 2001 - Fecha de aprobado: 19 de octubre de 2001

Solicitud de sobretiros: Dr. Héctor Samuel López Moreno. Departamento de Biomedicina Molecular, Centro de Investigación y de Estudios Avanzados del Instituto Politécnico N acional.Avenida Instituto Politécnico N acional N 0.2508, colonia San Pedro Zacatenco, Delegación Gustavo A. Madero, México, D.F., México. Correo electrónico: hlopez@ mail.cinvestav.mx 
a fase de activación de los linfocitos $\mathrm{T}$ vírgenes L $\mathrm{CD}^{+}{ }^{+}$o Th (del inglés "helper"), está dada por el reconocimiento de determinantes antigénicos presentados en el contexto de moléculas del complejo principal de histocompatibilidad de clase II (MHC-II), expresadas sobre células presentadoras de antígeno profesionales, como células dendríticas, linfocitos B y macrófagos. La especificidad de la respuesta inmune evocada por estos linfocitos $\mathrm{T} \mathrm{CD} 4^{+}$está dada por la expansión selectiva de las clonas capaces de reconocer dichos determinantes antigénicos, y de diferenciarse en células efectoras que contribuyen a la protección del organismo contra diferentes agentes infecciosos. Las parasitosis humanas continúan siendo uno de los principales problemas de salud en el mundo. No obstante el gran esfuerzo invertido por diferentes grupos de investigación aún no se dispone de una vacuna comercial contra ninguna parasitosis humana. Lo cual indica claramente el insuficiente entendimiento que se tiene acerca de los mecanismos inmunes protectores contra los parásitos, así como también ilustra los sofisticados mecanismos de evasión inmune de los parásitos. Ultimamente se han hecho avances muy importantes en el conocimiento de la respuesta inmune contra parásitos. Una de las observaciones clave para el control o el favorecimiento de las parasitosis es la polarización de la respuesta inmune de los linfocitos T CD4 ${ }^{+}$en Th1 o Th2. ${ }^{1}$ Esta clasificación se hizo de acuerdo con el patrón de citocinas producidas por estas células (figura 1). ${ }^{2}$ Los linfocitos Th1 secretan interferón $\gamma$ (IFN- $\gamma$ ), interleucina 2 (IL-2) y factor de necrosis tumoral beta (TNF- $\beta$ ). Estas citocinas participan en la activación de macrófagos y en la hipersensibilidad retardada. Por su parte, los linfocitos Th2 producen IL-4, IL-5, IL-6, IL-9, IL-10 e IL-13, cooperando con la respuesta inmune humoral a través de la proliferación de linfocitos B y del cambio de isotipo de los anticuerpos para la producción de inmunoglobulina $\mathrm{E}$ ( $\operatorname{IgE}$ ) e IgG1 (en ratón) o IgG4 (en humanos), y promoviendo la diferenciación de eosinófilos y mas-

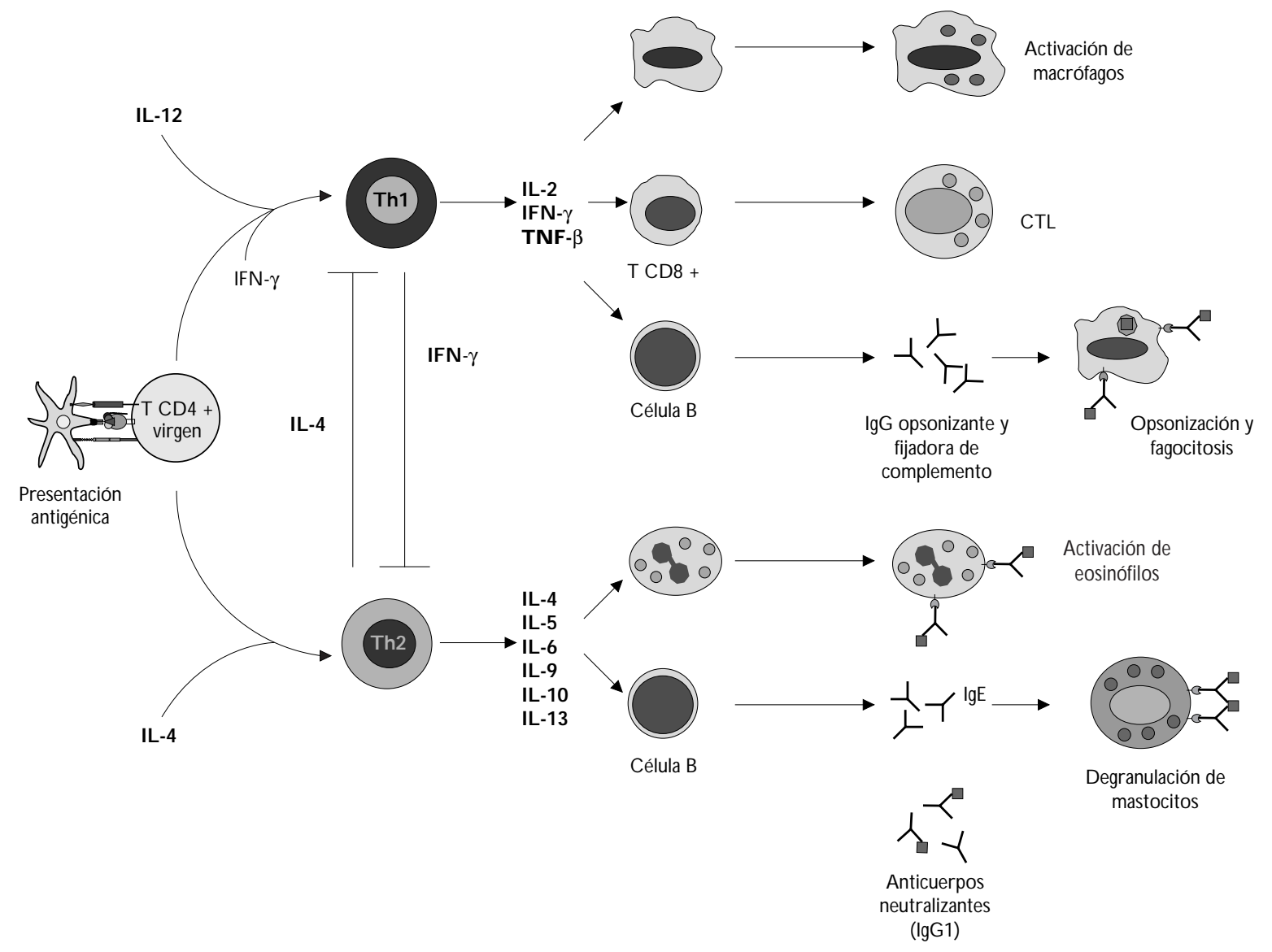

Figura 1. Funciones efectoras de las subpoblaciones T CD4+ (Th1 y Th2). Modificado de Abbas y COLABORADORES, $1996^{5}$ 
tocitos. Debido a estas funciones, el fenotipo Th2 se ha asociado con alergias. Existe otra subpoblación de linfocitos $\mathrm{T} \mathrm{CD} 4^{+}$que produce una mezcla de citocinas Th1/Th2 a la cual se le ha denominado Th0.3-7

Esta dicotomía de los linfocitos Th (Th1/Th2) se evidenció inicialmente en clonas de linfocitos $\mathrm{T} \mathrm{CD} 4^{+}$ de ratón, y posteriormente se demostró in vivo en ratones infectados con Leishmania major. ${ }^{1,8}$

En términos generales, la polarización de la respuesta inmune en Th1 o Th2 es más evidente en el caso de las parasitosis producidas por protozoarios, o bien por nemátodos intestinales en donde abundan ejemplos ampliamente documentados. ${ }^{1}$ No es el caso para las parasitosis tisulares, dentro de las cuales el Schistosoma ha sido ampliamente estudiado ${ }^{1}$ y se inicia así el estudio inmunológico de céstodos de los géneros Taenia y Echinococcus.

Taenia sp. y Echinococcus sp., pertenecientes a la clase Cestoidea, tienen un ciclo de vida que es muy similar (figura 2), ya que dependen de un hospedero intermediario que al ingerir sus huevecillos permite el desarrollo del estadio larvario, que puede ser un cisticerco o quiste hidatídico (con localización extraintestinal); el ciclo se completa cuando el hospedero intermediario es ingerido por el definitivo, en cuyo intestino se desarrolla el estadio adulto. Tanto para Taenia sp., como para Echinococcus sp., el humano es el hospedero intermediario accidental. ${ }^{9}$

Con el fin de elucidar la participación de las subpoblaciones de linfocitos $\mathrm{T}$ en la respuesta inmune

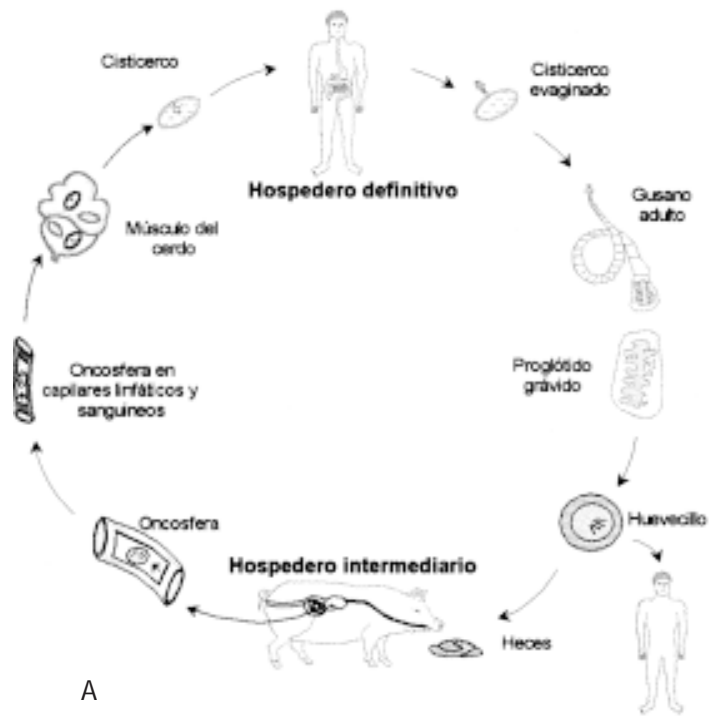

protectora o permisiva en cestodiasis tisulares (cisticercosis e hidatidosis), se han llevado a cabo diversos estudios en modelos experimentales y en humanos. La revisión de estos estudios es el objetivo del presente trabajo.

\section{Participación de los linfocitos T cooperadores $\mathrm{CD}^{+}$en cestodiasis tisulares}

\section{Cisticercosis}

Cisticercosis experimental

Los principales modelos experimentales empleados para el estudio de la cisticercosis son T. taeniaeformis en rata y ratón, y T. crassiceps en ratón. Otro modelo que también ha sido empleado es el de T. pisiformis en conejo; sin embargo, este modelo tiene algunas desventajas, ya que es sustancialmente más caro y no ofrece la homogeneidad genética que proporcionan las cepas de roedores. ${ }^{10}$

El modelo de cisticercosis por T. taeniaeformis asemeja en mucho a la infección natural. En este modelo se observó que la destrucción del estadio oncosferal del parásito, que previene el desarrollo de los cisticercos, está mediada principalmente por anticuerpos del subtipo IgG2a y complemento. ${ }^{10-12}$ Además, esta resistencia puede ser transferida pasivamente a roedores recipientes. ${ }^{11}$ Debido a dificultades metodológicas con

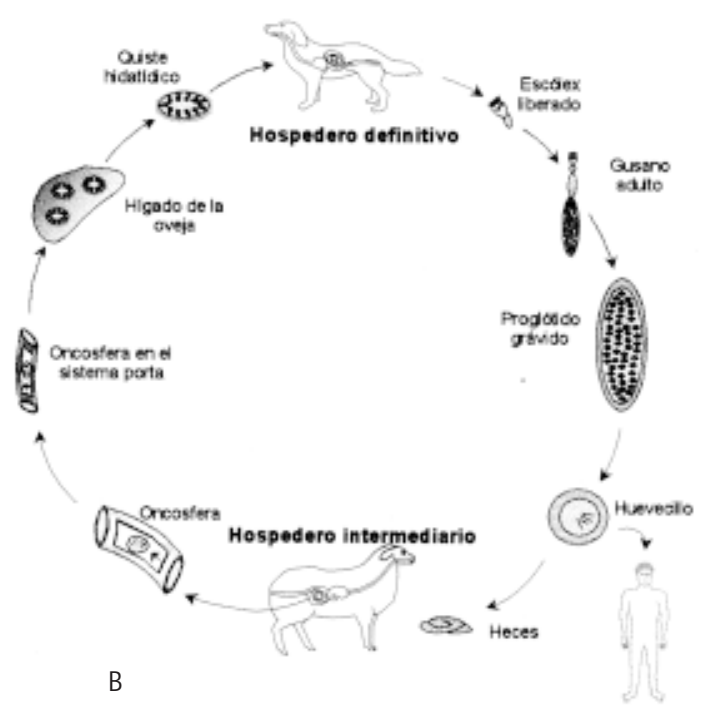

Figura 2. Ciclo de vida de céstodos del orden Ciclo phyllidea.A) Ciclo vital de Taenia solium. B) Ciclo vital de ECHINOCOCCUS SP 
el modelo de T. taeniaeformis, actualmente el modelo de cisticercosis por T. crassiceps en ratón es el más empleado para estudiar la participación de los linfocitos Th en esta parasitosis. ${ }^{13-18}$ La importancia de la respuesta inmune timo-dependiente se puso en evidencia al infectar ratones BALB/c timectomizados con cisticercos de $T$. crassiceps, observándose un gran incremento en la carga parasitaria respecto de ratones testigo. ${ }^{13}$ Estos resultados sugirieron la importancia de los linfocitos Th para limitar el desarrollo del cisticerco de T. crassiceps.

Diversos estudios se han orientado a caracterizar el fenotipo de la respuesta inmune Th1/Th2 con base en los perfiles de citocinas. ${ }^{14-19}$ En las etapas tempranas de esta infección experimental, alrededor de la primera semana posinfección (p.i.), el tipo de respuesta inmune predominante es Th1, la cual se asocia con un desarrollo limitado del parásito. ${ }^{15,16}$ A medida que la infección progresa, la respuesta inmune se polariza hacia un fenotipo Th2 con un incremento concomitante en la carga parasitaria. ${ }^{16}$ Hasta la fecha no se conocen con detalle los mecanismos que modulan el cambio en la subpoblación predominante; sin embargo, se postula que tanto factores del hospedero como del parásito podrían jugar un papel relevante. Lo anterior quedó confirmado por Terrazas y colaboradores en 1998, quienes demostraron que al tratar ratones infectados con IFN- $\gamma$ e IL-2 recombinantes, o con anticuerpos anti-IL-10, se incrementaba la resistencia a la parasitosis a las cuatro semanas p.i. ya que el número de cisticercos disminuía significativamente. ${ }^{17} \mathrm{En}$ analogía con lo que se ha descrito para el Schistosoma, en donde el cambio de Th1 a Th2 modula la progresión de los granulomas, Robinson y colaboradores en 1997 proponen que la respuesta Th1 podría jugar un papel muy importante, tanto en la patogénesis de la enfermedad como en la destrucción inicial del parásito. Toenjes y colaboradores en 1999 detectaron un incremento en la población de linfocitos $\mathrm{T} \gamma \delta$ en los exudados peritoneales de ratones infectados con cisticercos de T. crassiceps a partir de los 10 días p.i., lo que condujo a estos autores a pensar que esta población de linfocitos $\mathrm{T}$ podría tener un papel relevante en la cisticercosis por T. crassiceps. Sin embargo, cuando probaron su hipótesis empleando ratones deficientes para la cadena $\delta$, esta idea quedó descartada, ya que al evaluar los niveles séricos de las citocinas reguladoras de las subpoblaciones Th (IL-10, IL-4 e IFN- $\gamma$ ) así como el grado de carga parasitaria, no se encontraron diferencias respecto de los ratones de tipo silvestre. Lo anterior sugiere que esta población de linfocitos T no participa en la inmunidad contra el metacéstodo. ${ }^{18,19}$

Recientemente, Cardona y colaboradores describieron en 1999 un nuevo modelo de cisticercosis ex- perimental para estudiar la neurocisticercosis (NC). Este modelo se basó en la infección intracraneal de ratones BALB/c con el metacéstodo del parásito $\mathrm{Me}$ socestoides corti. Mediante cinéticas p.i. Cardona y colaboradores evaluaron la distribución del metacéstodo en el cerebro de los ratones infectados, las poblaciones celulares del sistema inmune presentes en el cerebro de estos ratones, así como los perfiles de citocinas que predominaban. Los resultados mostraron que a partir de la tercera semana p.i. más de $50 \%$ de los metacéstodos se localizaban en el parénquima. En tanto que, a partir de la primera semana p.i., la población de linfocitos $\mathrm{T}$ que predominaba en la región extraparenquimal o parenquimal, era del fenotipo $\mathrm{T} \gamma \delta$, cuyo repertorio está constituido predominantemente por

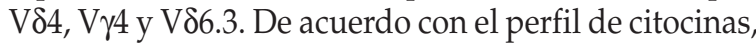
a partir de la primera semana p.i, el fenotipo predominante fue Th1, siendo esto más claro en la región extraparenquimal, donde se detectaron IL-2, IL-12, IL-15 e IFN- $\gamma$, mientras que sólo IL-12 e IFN- $\gamma$ fueron detectados en el parénquima aunque en menor proporción. A diferencia de lo que ocurre en el peritoneo, en este modelo predominaron los linfocitos $\mathrm{T} \gamma \delta$; no obstante, los autores no van más allá de la simple descripción de sus datos y omiten mencionar la integridad o el daño de los metacéstodos. Esta información podría ser complementada realizando estudios en ratones deficientes en linfocitos $\mathrm{T} \gamma \delta .^{20}$

\section{Cisticercosis humana}

Los datos que se tienen sobre la respuesta inmune celular en cisticercosis humana son escasos.

Del Brutto y colaboradores en 1991 tipificaron el patrón genético de HLA en un grupo de pacientes mexicanos mestizos con NC. Una de las diferencias más notables fue que los pacientes tenían una baja frecuencia del alelo DQw2 respecto del grupo testigo (4.2\% contra $31.7 \%) .{ }^{21}$ Ostrozky-Zeichner y colaboradores, en 1996, encontraron que las citocinas proinflamatorias IL-1 $\beta$ e IL-6 estaban aumentadas en muestras de líquido cefalorraquídeo (LCR) de pacientes con NC respecto del grupo testigo, lo cual no es sorprendente ya que sólo estudiaron formas activas de NC. ${ }^{22}$ Por su parte, Evans y colaboradores en 1998 encontraron un aumento en los niveles de IL-5 en LCR de pacientes con NC, comparado con el grupo testigo. El incremento de esta citocina en los pacientes sugiere que, al igual que en la cisiticercosis experimental, el fenotipo Th2 está relacionado con la permisión de la enfermedad. ${ }^{23}$ Restrepo y colaboradores en 1998 reportaron que la respuesta de linfocitos Th en pacientes con NC podría ser Th1. Estos resultados deben tomarse con reserva, ya que de los cuatro pacientes incluidos en el estudio, 
solamente a uno de ellos se le analizaron las citocinas de ambos fenotipos. ${ }^{24}$ Recientemente, Grewal y colaboradores publicaron un análisis de la respuesta Th en pacientes con NC utilizando células mononucleares de sangre periférica; no obstante que los autores proponen que la respuesta está polarizada hacia Th1, sus datos no son muy contundentes, ya que las concentraciones de las citocinas que evaluaron (IL-2, IL-4 e IFN- $\gamma$ ), en 15 pacientes con $\mathrm{NC}$, son muy similares a las obtenidas con el grupo testigo. ${ }^{25}$

\section{Hidatidosis}

\section{Hidatidosis experimental}

En hidatidosis también se han empleado modelos de ratón para estudiar la participación de los linfocitos Th; en este caso se tiene la ventaja de usar la misma especie que infecta al humano (E. multilocularis), lo cual no es posible en la cisticercosis.

La hidatidosis ha sido clasificada como primaria o secundaria de acuerdo con el desarrollo del metacéstodo. La hidatidosis primaria comprende las primeras seis o siete semanas p.i. en las cuales el quiste presenta un desarrollo lento. En la hidatidosis secundaria el quiste presenta un desarrollo acelerado a partir de la octava semana p.i. ${ }^{26}$ Estos datos sugieren que la respuesta inmune celular en los ratones con hidatidosis crónica puede estar polarizada hacia el fenotipo Th2, que permite el desarrollo del parásito in vivo. En uno de los primeros estudios se evaluó la hipersensibilidad retardada en ratones con hidatidosis primaria o secundaria, mediante la inflamación del cojinete plantar en respuesta al estímulo con un extracto crudo de E. multilocularis. Las diferencias principales se observaron a las 48 h, tiempo en el cual la inflamación había disminuido aproximadamente tres veces en ratones con hidatidosis secundaria respecto a los ratones con hidatidosis primaria. ${ }^{26}$ Estos datos sugieren que la respuesta Th1 puede estar disminuida en los ratones con hidatidosis crónica.

Con la finalidad de elucidar la participación de los linfocitos T y B en el desarrollo del quiste hidatídico se infectaron ratones con inmunodeficiencia severa combinada (SCID) y ratones C.B-17. Ambas cepas cuentan con el mismo fondo genético, pero los ratones SCID carecen de linfocitos T y B por tener defectuoso el gen que codifica para la enzima proteínacinasa dependiente del ácido desoxirribonucleico (del inglés "DNA-dependent protein kinase"). Los ratones SCID permitieron un mayor crecimiento del metacéstodo en la etapa secundaria respecto al grupo testigo (ratones C.B-17). En los ratones SCID los quistes llegaron a pesar hasta $17.5 \mathrm{~g}$ mientras que en los C.B17 el peso osciló alrededor de $1 \mathrm{~g} .{ }^{27}$ En 1992 Liance y colaboradores analizaron, en el curso de la infección con E. multilocularis en ratones de la cepa AKR, el efecto de la ciclosporina A (CsA), droga que unida a la ciclofilina inhibe la activación del linfocito $\mathrm{T}$ al bloquear la acción de calcineurina. Un grupo fue tratado con CsA al día 45 p.i., mientras que otro fue tratado con CsA antes de la infección y hasta el día del sacrificio (día 80). En el primer grupo la carga parasitaria prácticamente no se modificó respecto al grupo testigo infectado y no tratado, en cambio en el segundo grupo este parámetro aumentó significativamente. ${ }^{28} \mathrm{De}$ los estudios anteriores es posible concluir que los linfocitos T (y en particular Th1), y B tienen un papel fundamental para el control de la hidatidosis.

Emery y colaboradores en 1997 efectuaron un estudio orientado a definir el fenotipo de respuesta Th que confiere resistencia a la infección hidatídica secundaria, empleando cuatro cepas de ratones, las A/J, C57BL/6, DBA/1, y BALB/c; las dos primeras consideradas como las más resistentes y las dos últimas las más susceptibles. Estos autores demostraron que la resistencia al desarrollo de los parásitos en los ratones A/J se asoció con una fuerte respuesta proliferativa de esplenocitos antígeno-específicos in vitro, así como también con un incremento en la producción de IFN- $\gamma$ e IL-2 inducidos por los antígenos de E. multilocularis. En tanto que la susceptibilidad de los ratones BALB/c se asoció con altos niveles de IL-4..$^{29}$ Estos resultados les permitieron concluir que la resistencia a esta parasitosis está mediada por una respuesta inmune Th1. Lo anterior es consistente con los datos obtenidos en los modelos de cisticercosis experimental. ${ }^{16-18}$

Amiot y colaboradores en 1999 analizaron la participación de TNF- $\alpha$ y linfotoxina $\alpha(\mathrm{LT}-\alpha)$ en la equinococosis alveolar, generando ratones homocigotos deficientes en ambas citocinas. Al comparar la carga parasitaria de estos ratones deficientes para ambas citocinas, con la de ratones silvestres, observaron un incremento de este parámetro en los ratones deficientes. En muchos de los ratones silvestres las lesiones hepáticas involucionaron. Los parásitos muertos fueron "acordonados" por granulomas conformados por numerosos macrófagos y linfocitos que conducían a una fibrosis focal hepática en etapas tempranas de la infección. En contraste, muchos ratones deficientes albergaban metacéstodos mezclados con leucocitos, en abscesos purulentos con una amplia fibrosis irregular secundaria, en la etapa tardía de la infección. Este estudio muestra que TNF- $\alpha$ y LT- $\alpha$ tienen una participación esencial en los mecanismos de protección contra E. multilocularis. ${ }^{30}$ 


\section{Hidatidosis humana}

La hidatidosis humana se ha asociado con una disminución de la función de los linfocitos T in vitro. ${ }^{31}$ Profumo y colaboradores en 1994 analizaron la respuesta inmune celular antígeno específica en 18 pacientes con hidatidosis contra las subunidades del antígeno 5 y B de $E$. granulosus en células mononucleares de sangre periférica, posiblemente con la finalidad de delimitar la o las regiones correspondientes a los determinantes antigénicos para los linfocitos Th del antígeno 5. Dicho antígeno es una lipoproteína termolábil que presenta dos subunidades de 55 y $65 \mathrm{kDa}$ en geles de poliacrilamida que contienen dodecil sulfato de sodio (SDS-PAGE) en condiciones no reductoras. El antígeno $B$ es una lipoproteína termoestable que presenta tres subunidades de 12, 16 y $20 \mathrm{kDa}$ en SDS-PAGE en condiciones no reductoras y reductoras. ${ }^{32}$ Los resultados de estos autores muestran que la subunidad de $55 \mathrm{kDa}$ del antígeno 5 y la subunidad de $20 \mathrm{kDa}$ del antígeno $\mathrm{B}$ son las que inducen mayor respuesta proliferativa respecto a los testigos. ${ }^{31}$ Kharebov y colaboradores en 1997 también realizaron ensayos de proliferación celular antígeno específico empleando el antígeno B. Ellos analizaron las muestras de 41 pacientes, de los cuales $83 \%$ respondieron positivamente. No obstante, no ensayaron la antigenicidad de cada subunidad de este antígeno, por lo que su trabajo no hace ninguna aportación adicional a lo descrito por Profumo y colaboradores. ${ }^{33}$

Sturm y colaboradores en 1995 reportaron que la IL-5 era la citocina producida predominantemente por las células mononucleares de sangre periférica de pacientes con hidatidosis, por lo que la respuesta Th2 también estaría favoreciendo la enfermedad, como en el caso de la cisticercosis. ${ }^{23,34}$

Recientemente, Dreweck y colaboradores en 1999 analizaron algunas citocinas y quimiocinas producidas por células mononucleares de sangre periférica de pacientes, en respuesta al estímulo con metacéstodos vivos de E. multilocularis. Las quimiocinas que analizaron fueron la IL-8 y la proteína quimoatrayente de monocitos 1 (MCP-1), en tanto que las citocinas que evaluaron fueron la IL-12 e IL-13. Tanto las citocinas como las quimiocinas analizadas en este trabajo eran producidas mayormente por los leucocitos de pacientes comparado con el grupo testigo. Dreweck y colaboradores sugieren que estas citocinas relacionadas con respuestas tipo Th1 y Th2, así como las quimiocinas detectadas, pueden iniciar mecanismos de citotoxicidad dependiente de anticuerpos (ADCC, del inglés "antibody-dependent cellular cytotoxicity"), y que esta respuesta puede comprometer la progresión de la pa- rasitosis; $;{ }^{35}$ sin embargo, esta suposición no concuerda con ninguno de los trabajos descritos arriba, donde el fenotipo Th2 parece estar involucrado en la permisión de la hidatidosis. Es importante señalar que la ADCC es un mecanismo dependiente del fenotipo Th2, por lo que éste no parece ser el mecanismo efector contra el metacéstodo de Echinococcus.

\section{Conclusiones}

Los datos revisados en el presente trabajo sugieren que las condiciones inmunológicas más favorables para el desarrollo del cisticerco o del quiste hidatídico, en el humano y en el ratón, están asociadas con el tipo Th2. Por lo tanto, es posible concluir que el fenotipo que está relacionado con una respuesta inmune protectora o no permisiva para el desarrollo de estas cestodiasis es del fenotipo Th1. No obstante que prácticamente todos los trabajos sugieren la relevancia de la respuesta Th1 para el control de estas parasitosis, en ninguno de ellos se propone algún mecanismo efector para intentar esclarecer cómo este fenotipo podría combatir a estos parásitos. Además, ninguno de ellos describe algún determinante antigénico para linfocitos Th de estos parásitos, que pudiera estar participando en la polarización de la respuesta hacia un fenotipo permisivo (Th2), o bien, hacia un fenotipo protector (Th1). Por otro lado, se ha relacionado la disminución de los niveles séricos de testosterona en ratones machos BALB/c infectados con cisticercos de T. crassiceps, con un incremento en la carga parasitaria durante la etapa crónica p.i. De acuerdo con Morales y colaboradores, ello pudiera deberse a una posible desregulación del fenotipo Th1, 36,37 a pesar de que no muestran formalmente cambios del patrón de linfocitos Th como se han descrito en el modelo de infección por Plasmodium chabaudi, donde se ha observado que la testosterona participa en la regulación de Th1..$^{38}$

Aunque no se ha propuesto un mecanismo efector contra el cisticerco o el quiste hidatídico, es posible que la secuencia de eventos que conducen a la destrucción de ambos metacéstodos sea muy similar. Retomando los estudios histopatológicos de la cisticercosis porcina, que han permitido clasificar las etapas a través de las cuales se lleva a cabo la destrucción del metacéstodo, descritas por de Aluja y Vargas en 1988, ${ }^{39}$ la secuencia de eventos podría ser la siguiente: en las etapas tempranas la "primera línea de ataque" está formada principalmente por eosinófilos; sin embargo, contrario a lo descrito para diferentes nemátodos y algunas especies de Schistosoma, ${ }^{40}$ al cisticerco aparentemente no le ocasionan daños importantes. En la etapa tardía, cuando el tegumento del parásito empieza a 
degenerarse se coincide con la presencia de macrófagos, los cuales se alinean en forma de palizadas (células epitelioides) alrededor del metacéstodo; en estas etapas se observa un incremento en los agregados de linfocitos donde los $\mathrm{T}$ presentes pueden diferenciarse a Th1 por la IL-12 producida por los macrófagos y, a su vez, estos linfocitos Th1 pueden activar a otros macrófagos presentes en el microambiente, mediante la producción de IFN- $\gamma$. Finalmente, los macrófagos activados pueden secretar factores de crecimiento de células mesenquimatosas, favoreciendo de esta manera la fibrosis que se observa en la etapa final, posterior a la destrucción del metacéstodo.

Será necesario continuar con los estudios con el fin de elucidar con precisión los mecanismos efectores de la respuesta inmune Th involucrados en la eliminación de estos céstodos, dentro de lo cual la identificación de los determinantes antigénicos será un componente fundamental.

\section{Agradecimientos}

Agradezco de manera muy especial a la Dra. Patricia Talamás Rohana por su valiosa asesoría y apoyo en la escritura del presente trabajo, así como también por sus críticas y comentarios. Agradezco a la Dra. Dolores Correa Beltrán por sus sugerencias.

\section{Referencias}

1. Pearce $E$, Scott $P$, Sher $A$. Immune regulation in parasitic infection and disease. En: Paul W, ed. Fundamental immunology. 4a edición. México, D.F.: Lippincott-Raven, 1999:1271-1294.

2. Mossman T, C herwinsky H, Bond M, G ieldlin M, C offman R. Two types of murine helper $T$ cell clone. I. D efinition according to profiles of lymphokine activities and secreted proteins. J Immunol 1986;136:2348-2357.

3. 0 'GarraA. Cytokines induce the develo pment of funcionally heterogeneous T helper cell subsets. Immunity 1998:8:275-283.

4. Constant $\mathrm{S}$, Bottomly K. Induction of Th1 and Th2 CD 4 ${ }^{+} \mathrm{T}$ cell responses:The alternative approaches. Annu Rev Immunol 1997;15:297-322.

5.A bbas A, Murphy K, Sher A. Funcional diversity of helper T lymphocytes. Nature 1996;383:787-793.

6. Romagnani S. Lymphokine production by human T cells in disease states. Annu Rev Immunol 1994;12:227-257.

7. Reiner S, Locksley R. The worm and the protozoa: Stereotyped responses or distintic antigens? Parasitol Today 1993;9:258-260.

8. Sher A, Gazzinelli R, 0 swald I, C lerici M, Kullberg M, Pearce E et al. Role of T-cell derived cytokines in the downregulation of immune responses in parasitic and retroviral infection. Immunol Rev 1992;127:183-203.

9. Beck W, D avies J. Parasitología médica. 3a. edición. México, D.F.: Interamericana, 1984

10.W illiams J, Engelkirk P, Lindsay M. Mechanisms of immunity in rodent cysticercosis. En: Flisser A, W illms K, Laclette J, ed. Cysticercosis: Present state of knowledge and perspectives. N ueva York (NY):Academic Press, 1982:621-632.
11. Leid R,W illiams J.The immunological response of the rat to infection with Taenia taeniaeformis. Immunology 1974;27:195-207.

12. Mitchell G. Genetic variation in resistance of mice to Taenia taeniaeformis:A nalysis of host-protective immunity and immune evasion. En:Flisser A, W illms K, Laclette J, ed. Cysticercosis: Present state of knowledge and perspectives. N ueva York (N Y):A cademic Press, 1982:575-584.

13. Bojali R, Terrazas L, GovezenskyT, Sciutto E, Larralde C. Thymus-related cellular immune mechanisms in sex-associated resistance to experimental murine cysticercosis (Taenia crassiceps).J Parasitol 1993;79:384-389. 14.Villa 0 , Kuhn R. Mice infected with the larvae of Taenia crassiceps exhibit aTh2-like immune response with concomitant anergy and downregulation of Th1-associated phenomena. Parasitology 1996;112:561-570.

15. Robinson $P$, Atmar R, Lewis D,W hite C. G ranuloma cytokines in murine cysticercosis. Infect Immun 1997;65:2925-2931.

16. Terrazas L, Bojalil R, Govezensky T, Larralde C. Shift from an early protective Th1-type immune response to a late permisiveTh2-type response in murine cysticercosis (Taenia crassiceps). J Parasitol 1998;84:74-81.

17. Terrazas L, Cruz M, Rodríguez-Sosa M, Bojalil R, García-Tamayo F, Larralde $C$.Th1-type cytokines improve resistance to murine cysticercosis caused by Taenia crassiceps. Parasitol Res 1999;85:135-141.

18. Toenjes S, Spolski R, Mooney K, Kuhn R. The systemic immune response of BALB/C mice infected with larval Taenia crassiceps is a mixed Th1/ Th2-type response. Parasitology 1999;118:623-633.

19. Toenjes S, Spolski R, Mooney K, Kuhn R. $\gamma \delta$ T cells do not play a major role in controlling infection in experimental cysticercosis. Parasitology 1999;119:413-418.

20. Cardona A, Restrepo B, Jaramillo J, Tale J. D evelopment of an animal model for neurocysticercosis: Immune response in the central nervous system is characterized by a predominance of $\gamma \delta \mathrm{T}$ cells. J Immunol 1999;162:995-1002.

21. Del Brutto 0, G ranados G,Talamás O, Sotelo J, Go rodezky C. Genetic pattern of the HLA system: HLA A, B, DR and DQ antigens in Mexican patients with parenchymal brain cysticercosis. Hum Biol 1991;63:85-93.

22. 0 strosky-Zeichner L, García-Mendoza E, Ríos C, Sotelo J. Humoral and cellular immune response within the subarachnoid space of patiens with neurocysticercosis. Arch Med Res 1996;27:513-517.

23. Evans C, García H, Hartnell A, Gilman R, José P, Martínez M et al. Elevated concentrations of eotaxin and interleukin-5 in human neurocysticercosis. Infect Immun 1998;66:4522-4525.

24. Restrepo B, Llaguno P, Sandoval M, Enciso J, Teale J. A nalysis of immune lesions in neurocysticercosis patients: Central nervous system response to helminth appears Th1-like instead of Th2.J N euroimmunol 1998:89: 64-72.

25. Grewal J, Kaur S, Bhatti G, Singh I, G anguly N , Mahajan R et al. C ellular immune responses in human neurocysticercosis. Parasitol Res 2000;86: 500-503.

26.Ali-Khan Z. Echinococcus multilocularis: Cell-mediated immune response in early and chronic alveolar murine hydatidosis. Exp Parasitol 1978;46: 157-165.

27. Playford M, O oi H, O kuY, Kamiya M. Secondary Echinococcus multilocularis infection in severe combined immunodeficient (SCID) mice: Biphasic growth of the larval cyst mass. Int J Parasitol 1992;22:975-982.

28. Liance M, Bresson-Hadni S, Vuitton D, Lenys D, C arbillet J, Houin R. Effects of cyclosporin A on the course of murine alveolar echinococcosis and on specific cellular and humoral immune responses against Echinococcus multilocularis. Int J Parasitol 1992;22:23-28.

29. Emery I, Liance M, Leclerc C. Secondary Echinococcus multilocularis infection in A/J mice: D elayed metacestode development is associated with Th1 cytokine production. Parasite Immunol 1997;19:493-503.

30. A miot F,Vuong P, D efontaines M, Pater C, D autry F, Liance M. Secondary alveolar echinococcosis in lymphotoxin- $\alpha$ and tumor necrosis factor- $\alpha$ deficient mice: Exacerbation of Echinococcus multilocularis larval growth is associated with cellular changes in the periparasitic granuloma. Parasite Immunol 1999;21:475-483. 
31. Lightowlers M. Immunology and molecular biology of Echinococcus infections. Int J Parasitol 1990;20:471-478.

32. Profumo E, O rtona E, Rigano R, G ioia I, N otargiacomo S, loppolo S et al. Cellular and humoral responses to antigenic subunits of Echinococcus granulosus cyst fluid in hydatid patients. Parasite Immunol 1994;16: 393398.

33. Kharebov A, N ahmias J, El-on J. Cellular and humoral immune responses of hydatidosis patients to Echinococcus granulosus purified antigens.Am JTrop Med Hyg 1997;57:619-625.

34. Sturm D, Menzel J, Gottstein B, Kern P. Interleukin-5 is the predominant cytokine produced by peripheral blood mononuclear cells in alveolar echinococcosis. Infect Immun 1995;63:1688-1697.

35. D reweck C, Soboslay P, Schulz-Key H, Gottstein B, Kern P. Cytokine and chemokine secretion by human peripheral blood cells in response to viable Echinococcus multilocularis metacestode vesicles. Parasite Immunol 1999:21:433-438.

36. Morales-Montor J, Rodríguez-D orantes M, Mendoza-Rodríguez A, Camacho-Arroyo I, Cerbón M. Differential expression of the estrogen-regu- lated proto-oncogenes c-fos, $\mathrm{c}$-jun, and bcl-2 and of the tumor-suppressor p53 gene in the male mouse chronically infected with Taenia crassiceps cysticerci. Parasitol Res 1998;84:616-622.

37. Morales-Montor J, Rodríguez-D orantes M, Cerbón M. Modified expression of steroid $5 \alpha$-reductase as well as aromatase, but not cholesterol side-chain cleavage enzyme, in the reproductive system of male mice during (Taenia crassiceps) cysticercosis. Parasitol Res 1999;85:393-398.

38. Z hang Z, C hen L, Saito S, Kanagawa O , Sendo F. Possible modulation by male sex hormone of Th1/Th2 function in protection against Plasmodium chabaudi chabaudi AS infection in mice. Exp Parasitol 2000;96:121-129.

39. De Aluja S, Vargas $G$. The histopathology of porcine cysticercosis.Vet Parasitol 1988;28:65-77.

40. Behm C, $O$ vington $\mathrm{K}$. The role of eosinophils in parasitic helminth infections: Insights rom genetically modified mice. Parasitol Today 2000;16:202-209. 